\title{
AN ACCOUNT
}

\section{OF THE \\ ANASTOMOSIS OF THE ARTERIES}

\section{AT THE GROIN.}

\section{BY ASTLEY COOPER, EsQ. F.R.S.}

\author{
SURGEON TO GUY'S HOSPITAL.
}

Read July 13, 1813.

IN a paper which I had formerly the honour of reading to this Society, I. endeavoured to describe the course of the new channels for the blood, when the femoral artery has been obliterated by the operation for popliteal aneurism. Since that period I have had an opportunity of dissecting two persons in whom the iliac artery had been tied; and, as one of these had survived the operation a much longer time than the other, an opportunity was given, not only of seeing the blood-vessels, when the course of the blood is established, but also of tracing the gradual progress of the new circulation.

Hypothesis would lead to a belief that anastomosing vessels would be numerous in proportion to the time which had elapsed from the operation, but the reverse of this is the fact, for at first many vessels convey the blood originally conducted by 
the principal artery; but gradually the number of these channels becomes diminished, and, after a length of time, a few vessels conveniently situated for the new circulation, are becoming so much enlarged as to be capable of conveying an equal portion of blood to that which passed through the original trunk.

In examining, therefore, the two limbs, which I have the honour of shewing to the Society, many more anastomosing vessels are enlarged in that which had been recently the subject of the operation, than in the limb on which the operation had been performed for more than two years, a circumstance which has not arisen from a more successful injection, as the one had been as well injected as the other.

It may be further observed, that a person who has his iliac artery tied for an aneurism of the groin, recorers the use of the limb much more quickly than when the aneurism is situated in the middle of the thigh, for in the inguinal aneurism the principal anastomosing vessels are left free from pressure, but the femoral aneurism is buried so deeply in the muscles of the thigh, that the branches of the atteria profunda are compressed, and the passage of the blood to the lower part of the limb is impeded.

In about six weeks from the operation in the 
former case, the patient is able to make use of the limb, but in the latter the muscles of the leg and foot will be some months before they recover their powers, requiring the absorption of the aneurismal contents, and the consequent removal of the pressure upon the nerves and vessels.

This observation, however, applies principally to large aneurisms, on which account it is desirable in femoral aneurism, if not, indeed, in all others, to perform the operation in an early state of the disease.

One of these cases was that of a man of the name of Garrett Riley, who was a patient in Guy's Hospital, and had his iliac artery tied on the 14th of February, 1811; this man died ten weeks and six days after the operation, in consequence of the bursting of an aneurism at the bifurcation of the aorta; he was sitting, as I was informed, by his dresser, Mr. Barraud, in the square of the Hospital, when he suddenly fainted; he was taken into his ward, and in a few minutes afterwards expired.

Upon inspection of his body, beside the aneurism at the bifurcation which had burst, five aneurismal swellings will be seen in the limb, which I have now the honour to exhibit to the Society, one at the origin of 'the arteria profunda in the groin, a second in the middle of the thigh where 
the artery pierces the tendon of the triceps, which aneurism was of large size, and was that for which the operation was performed; a third aneurism was placed in the ham, and between the popliteal and femoral there were two smaller aneurisms.

This man was a bricklayer's labourer, and the great exertions he had made in carrying loads up ladders, was, in his mind, the cause of the dis. ease. Upon endeavouring to ascertain the mode in which the blood took its course through the limb, it was found that the femoral, tibial and fibular arteries were still open, and that the blood was conveyed into the femoral artery by the following anastomosis : The internal pudendal artery formed several large branches upon the side of the bulb of the penis, and these branches freely communicating with the external pudendal artery, had determined the blood into that artery, and by this channel into the femoral; the lateral sacral artery also sent a branch on the iliacus internus muscle, into the femoral artery, and the ilio lumbar artery freely communicated with the circumflex ilii, so that by these three routes, the blood found direct ingress to the femoral artery.

Numerous branches of arteries also passed from the lateral sacral to the obturator and epigastric arteries, the obturator in this case having its origin from the epigastric. 
Beside these arteries a free communication existed between the arteria profunda and circumflex arteries with the branches of the internal iliac; first, the gluteal artery sent a branch under the gluteus medius muscle to the external circumflex artery; secondly, the ischiatic artery gave two sets of branches of communication, one upon the gluteus maximus muscle to the arteria profunda, and another upon the sciatic nerve to the internal circumflex artery; the internal pudendal artery also sent a branch of communication to the internal circumflex; lastly, the obturator freely communicated with the internal circumflex.

These then are the channels for the blood in an early date from the operation, but at more remote periods, as the anastomosing branches become larger, they are less numerous, and the description of their course is much more simple.

The second case was that of James Nutter, aged 37, who had the operation of tying the iliac artery performed on the 24th August, 1810, on account of a large femoral aneurism above the tendon of the triceps. This man survived the operation nearly three years, and dying in Guy's Hospital, I had an opportunity of examining his body, and of learning the condition of the parts which had been the subject of the operation. The external iliac and the femoral arteries were 
obliterated, excepting about an inch of the femoral artery just below Poupart's ligament, which still remained open, and continued to convey a portion of the blood, but below this part it had become simply a ligamentous chord. The internal iliac artery sent first a very large artery of communication to the epigastric and obturator artery, so that the epigastric was supplied with blood from the internal iliac: secondly, the internal iliac sent an artery of communication upon the sciatic nerve to the internal circumflex artery. The gluteal artery gave a large branch to the origin of the profunda : lastly, the internal pudendal artery largely anastomosed with the obturator: the obturator, therefore, sprang in this case from two new sources, viz. from the internal iliac and from the internal pudendal artery, and the obturator, thus formed, sent two branches of communication to the internal circumflex artery. The arteria profunda was in this case supplied from two sources directly from the gluteal, and more indirectly from the internal circumflex by the obturator and ischiatic arteries. The external iliac artery was obliterated to the origin of the internal iliac, as other arteries usually are when ligatures are made upon them to the first large anastomosing vessel*. The principal

- I have been informed that Mr. George Bell, of Edinburgh, has a preparation of a limb, in which he had divided the femoral artery for poptiteal aneurism, and that the obliteration of the artery has not extended to the arteria profunda, as usually happens.

VOL. IV. 
agents then of the new circulation are the gluteal artery with the external circumflex, the obturator artery with the internal circumflex, and the ischiatic with the arteria profunda, and the obturator artery is supplied with blood principally by the internal pudendal when the obturator arises from the epigastric artery.

The iliac artery has now been so frequently tied for aneurism of the femoral artery at the groin, that no useful purpose can result from the narrative of a case of that kind, offering no uncommon circumstances. In Mr. Abernethy's works, and in a book published by Mr. Freer, the first and best histories of this operation are given. But in the two following cases the disease had proceeded to an extent to make the probability of success but small, and the result will shew that the operation may be successfully performed under the most adverse circumstances.

William Cowles, aged 37, came to London from Beccles, in Suffolk, on account of an aneurism in the right groin, which he attributed to his having walked five miles with a heavy burden upon his back six months ago, and a fortnight before the appearance of the aneurism. Journeying to London upon the top of a coach, a distance of more than one hundred miles, he' fell asleep upon his 
face, and pressing upon the tumour, he observed its surface changed to a gangrenous colour. When he was admitted into Guy's Hospital, the skin was purple in some parts, red in others, and extremely thin. It was obvious no time was to be lost, and the operation was performed upon the day of his admission, viz. the 22d of June, 1808 : two ligatures were made upon the iliac artery, and the vessel divided between them.

Nothing unusual occurred until the 30th of June, at ten o'clock at night, when a discharge of darkcoloured blood took place from the aneurismal sac, and the swelling became quid flaccid. I ordered a sponge dipped in vinegar and water to be applied upon the swelling.

In the six following days the skin from the surface of the sac sloughed, so that the aneurismal cavity was completely opened. July the 8 th, the upper ligature separated, and on the 9th the lower came away.

The wound then looked well, the aneurismal sac granulated; but the man's health beginning to fail, it was necessary to remove him about a mile out of London, where he gradually recovered. This man, who now resides in the neighbourhood of Beccles; was in London this year, 1813, perfectly well. 


\section{CASE II.}

April 30, 1818.

William Martin, aged 27, was admitted into Guy"s Hospital under the care of Mr. Forster, for a tracture of the olecranon, and after having been in the hospital three weeks, he requested his dresser (Mr. Johnson) to examine a swelling in the left groin, which proved a femoral aneurism, seated at a small distance below Poupart's ligament, and the integument over it was in a mortified state, being of a dark colour, and having three vesicles upon its surface.

As it was thought that an operation was immediately necessary, and Mr. Foster was not at the hospital, I was requested to see him. The man said that the swelling had been growing nearly twelve months, and had a pulsation when he first observed it. He attributed its commencement to an attempt to raise $3 \frac{1}{2} \mathrm{cwt}$. about three weeks before he first observed it.

The mortified state in which it was, he attributed to walking the distance of four miles three days before it was shewn to his dresser.

The tumour was as large as an orange; the pulsation in it very strong; its most projecting 
part was livid, and the surrounding parts of a deep red colour.

It appeared to me that no time was to be lost, and I immediately proceeded to tie the iliac artery. It may not be improper to remark, that the incision which I make for this purpose, is different to that usually advised ; for I begin it just above the abdommal tings, and carry it half an inch above Poupart's ligament, in a semilunar direction to one inch upon the inner side of the anterior and superior spinous process of the ilium.

Two ligatures were applied upon the artery, and the vessel divided between them.

\section{The ligatures separated on the 17th day.}

On the $23 \mathrm{~d}$ day after the operation, an incision was made into the aneurism, through the eschar, and the coagulated blood was discharged: the opening from the artery into the sac was very visible, but there was no bleeding from it.

The wound continued in a sloughy state for about three weeks, and then began to granulate; and although the restorative process went on slowly, the man was, after several weeks, discharged from the Hospital, completely cured, no lameness remaining. 